\title{
Mathematical modelling, determination and analysis of the thermostressed state in a thermosensitive three-layer hollow cylinder subjected to the convective-radiative heating
}

\author{
Kushnir R. M. ${ }^{1}$, Popovych V.S. ${ }^{1}$, Rakocha I. I. ${ }^{2}$ \\ ${ }^{1}$ Pidstryhach Institute for Applied Problems of Mechanics and Mathematics \\ of the National Academy of Sciences of Ukraine \\ $3 b$ Naukova str., 79060, Lviv, Ukraine \\ ${ }^{2}$ Lviv Polytechnic National University \\ 12 S. Bandera str., 79013, Lviv, Ukraine
}

(Received 1 December 2015)

\begin{abstract}
The stationary temperature distribution in a three-layer infinitely-long hollow cylinder is modeled and determined under the condition that the internal heat sources are distributed within the second layer in accordance to the parabolic law and the convective-radiative and convective heat exchange with the environment occurs on the inner and outer surfaces, respectively. The components of the thermostressed state were found and the effect, caused by the dependence of thermophysical and mechanical material properties on the temperature, along with the intensity of radiative heat exchange, in the distribution of the temperature and stress-strain state is analyzed.
\end{abstract}

Keywords: piecewise homogeneous cylinder, hollow cylinder, thermosensitive material, temperature, thermal stress

2000 MSC: $97 \mathrm{M} 50$

UDC: 593.3

\section{Introduction}

A wide class of modern structure members is presented by the multilayer assemblies of a rectilinear, cylindrical or spherical shape. Due to the fact that such structures are usually supposed to undergo the high- or lowtemperature heating under coacting force loading, the dependences of their thermophysical and mechanical properties on the temperature appear to be critical [1-5] for the adequate determination of the stress-strain state [1,3], which, in turn, is quite important for the prediciton of their durability and reliability.

By making use of the technique suggested in [1], a nonlinear mathematical model for the distribution of temperature in a three-layer (with respect to the radial coordinate) thermosensitive hollow cylinder is formulated. It is assumed that the second (intermediate) layer of the cylinder contains internal heat sources, which are distributed in accordance to the parabolic law. The inner and outer surfaces of the cylinder are exposed to the convective-radiative and convective heat exchange, respectively. On the layer interfaces, the conditions of perfect thermal contact are imposed. We have managed to determine the components of the stress-stain state induced by the determined non-uniform distribution of the temperature. The effect of the material thermosensitivity, along with the intensity of the radiative heat exchange, in the temperature and stress-strain state are analyzed.

This treatise is partially financed under a joint research project by the National Academy of Sciences of Ukraine and RFFI (the state registration number 0114U005081) 


\section{Mathematical model of the thermal conductivity of temperature field}

Consider an infinitely-long hollow cylinder consisting of three cylindrical layers made of dissimilar thermosensitive materials. Assume the cylinder to be heated by internal heat sources which are distributed within the intermediate layer due to the parabolic law

$$
W^{(2)}(r)=\frac{4 W_{0}^{2}}{\left(r_{3}-r_{2}\right)^{2}}\left(r-r_{2}\right)\left(r-r_{3}\right) .
$$

The inner, $r=r_{1}$, and outer, $r=r_{4}$, surfaces of the cylinder are subjected to the convective-radiative (with the thermal conductivity $\alpha_{1}$ and emissivity rate $\varepsilon_{1}$ ) and convective (with the thermal conductivity $\alpha_{3}$ ) heat exchange with the inner and outer surroundings of temperature $t=t_{\text {ins }}$ and $t=t_{\text {outs }}$, respectively. The perfect thermal contact is assumed on the layer interfaces $r=r_{2}$ and $\mathrm{r}=r_{3}$. Our objective is to determine the temperature distribution along with the components of stress-strain state in the cylinder under the foregoing conditions.

The mathematical model for the determination of temperature in the considered thermosensitive hollow cylinder is governed by:

- the heat-conduction equations for each layer

$$
\frac{1}{r} \frac{d}{d r}\left(r \lambda_{t}^{(i)} \frac{d t_{i}}{d r}\right)=W^{(i)}(r) \delta_{i 2}, \quad r_{i}<r<r_{i+1}, \quad i=\overline{1,3}
$$

- the boundary conditions on the inner and outer limiting surfaces

$$
\begin{gathered}
{\left[\lambda_{t}^{(1)}\left(t_{1}\right) \frac{d t_{1}}{d r}-\alpha_{1}\left(t_{1}-t_{\text {ins }}\right)-\sigma \varepsilon_{1}\left(t_{1}^{4}-t_{\text {ins }}^{4}\right)\right]_{r=r_{1}}=0,} \\
{\left[\lambda_{t}^{(3)}\left(t_{3}\right) \frac{d t_{3}}{d r}+\alpha_{3}\left(t_{3}-t_{\text {outs }}\right)\right]_{r=r_{4}}=0}
\end{gathered}
$$

- the interface contact conditions

$$
\left.t_{i}\right|_{r=r_{i+1}}=\left.t_{i+1}\right|_{r=r_{i+1}},\left.\quad \lambda_{t}^{(i)}\left(t_{i}\right) \frac{d t_{i}}{d r}\right|_{r=r_{i+1}}=\left.\lambda_{t}^{(i+1)}\left(t_{i+1}\right) \frac{d t_{i+1}}{d r}\right|_{r=r_{i+1}}, \quad i=1,2,
$$

where $\delta_{i j}=\left\{\begin{array}{ll}1, & i=j ; \\ 0, & i \neq j\end{array}\right.$ is the Kronecker delta and $\sigma$ stands for the Stefan-Boltzmann constant.

Model (1)-(4) is the nonlinear one due to the nonlinearity of the condition (2) and assumed dependence of the heat-conduction coefficients $\lambda_{t}^{(i)}\left(t_{i}\right)(i=\overline{1,3})$ on temperature. In the literature, such dependencies are usually given in a tabular form. In order to implement the theoretical analysis, it is desirable to have the heat-conduction coefficients in the form of explicit analytical expressions $\lambda_{t}^{(i)}=$ $=\lambda_{t}^{(i)}(t)$. To obtain such expressions, the tabular data are usually approximated with an analytical function by making use, e.g., of the least squares method.

Let the heat-conduction coefficients for each layer be given within the temperature range $\left[t_{b}, t_{e}\right]$. In what follows, we will describe them with the linear dependencies $\lambda_{t}^{(i)}=a_{i} t_{i}+b_{i}$, which is quite common. Then the unknown parameters $a_{i}$ and $b_{i}$ can be found by means of the least squares method in order to achieve the minimum difference between the sums of experimental and theoretical data.

Let us represent the heat-conduction coefficients of each layer in the form $\lambda_{t}^{(i)}\left(t_{i}\right)=\lambda_{t 0}^{(i)} \lambda_{*}^{(i)}\left(T_{i}\right)$, where $\lambda_{t 0}^{(i)}$ are constants in dimension of the heat-conduction coefficient, $\lambda_{*}^{(i)}\left(T_{i}\right)$ are dimensionless functions of dimensionless temperature $T_{i}=t_{i} / t_{e}, i=\overline{1,3}, t_{e}$ is the reference temperature. Due to such linear dependence of the heat-conduction coefficients on the temperature, such representations will have the following form:

$$
\lambda_{t}^{(i)}\left(t_{i}\right)=\lambda_{t 0}^{(i)}\left(1+k_{i}\left(T_{i}-T_{b}\right)\right),
$$


where $T_{b}=t_{b} / t_{e}, k_{i}=a_{i} t_{k} /\left(a_{i} t_{b}+b_{i}\right)$, and $\lambda_{t 0}^{(i)}=a_{i} t_{b}+b_{i}$.

By introducing a characteristic length value $l_{0}$ and the dimensionless coordinate $\rho=r / l_{0}$, mathematical model (1)-(4) can be given in the dimensionless form:

$$
\begin{gathered}
\frac{d}{d \rho}\left(\rho \lambda_{*}^{(i)}\left(T_{i}\right) \frac{d T_{i}}{d \rho}\right)=W^{(i)}(\rho) \delta_{2 i}, \quad \rho_{i}<\rho<\rho_{i+1}, \quad i=\overline{1,3} \\
{\left[\lambda_{*}^{(1)}\left(T_{1}\right) \frac{d T_{1}}{d \rho}-B i_{1}\left(T_{1}-T_{i n s}\right)-S k_{1}\left(T_{1}^{4}-T_{\text {ins }}^{4}\right)\right]_{\rho=\rho_{1}}=0,} \\
{\left[\lambda_{*}^{(3)}\left(T_{3}\right) \frac{d T_{3}}{d \rho}+B i_{3}\left(T_{3}-T_{\text {outs }}\right)\right]_{\rho=\rho_{4}}=0,} \\
\left.T_{i}\right|_{\rho=\rho_{i+1}}=\left.T_{i+1}\right|_{\rho=\rho_{i+1}},\left.\quad \lambda_{*}^{(i)}\left(T_{i}\right) \frac{d T_{i}}{d \rho}\right|_{\rho=\rho_{i+1}}=\left.K_{\lambda}^{(i+1)} \lambda_{*}^{(i+1)}\left(T_{i+1}\right) \frac{d T_{i+1}}{d \rho}\right|_{\rho=\rho_{i+1}}, \quad i=1,2,
\end{gathered}
$$

where $W^{(2)}(\rho)=-4 P o \rho\left(\rho-\rho_{2}\right)\left(\rho-\rho_{3}\right) /\left(\rho_{3}-\rho_{2}\right)^{2}, P o=W_{0}^{(2)} l_{0}^{2} /\left(\lambda_{t 0}^{(2)} t_{e}\right)$ is the Pomerantsev number, $B i_{i}=\alpha_{i} l_{0} / \lambda_{t 0}^{(i)}(i=1,3)$ denotes the Biot numbers, $S k_{1}=\sigma \varepsilon_{1} t_{e}^{3} l_{0} / \lambda_{t 0}^{(1)}$ stands for the Stark number, $K_{\lambda}^{(j)}=\lambda_{t 0}^{(j)} / \lambda_{t 0}^{(j-1)}(j=2,3), T_{\text {ins }}=t_{\text {ins }} / t_{e}, T_{\text {outs }}=t_{\text {outs }} / t_{e}$.

\section{Determination of the temperature field}

Let us introduce the Kirchhoff variables

$$
\theta_{i}=\int_{T_{b}}^{T_{i}} \lambda_{*}^{(i)}\left(T_{i}\right) d T_{i}, i=\overline{1,3},
$$

instead of dimensionless temperatures $T_{i}$. Then problem (6)-(9) can be reduced to the following one:

$$
\begin{gathered}
\frac{d}{d \rho}\left(\rho \frac{d \theta_{i}}{d \rho}\right)=W^{(i)}(\rho) \delta_{i 2}, \quad \rho_{i}<\rho<\rho_{i+1}, \quad i=\overline{1,3}, \\
{\left[\frac{d \theta_{1}}{d \rho}-B i_{1}\left(T_{1}\left(\theta_{1}\right)-T_{\text {ins }}\right)-S k_{1}\left(T_{1}\left(\theta_{1}\right)^{4}-T_{i n s}^{4}\right)\right]_{\rho=\rho_{1}}=0,} \\
{\left[\frac{d \theta_{3}}{d \rho}+B i_{3}\left(T_{3}\left(\theta_{3}\right)-T_{\text {outs }}\right)\right]_{\rho=\rho_{4}}=0,} \\
\left.T_{i}\left(\theta_{i}\right)\right|_{\rho=\rho_{i+1}}=\left.T_{i+1}\left(\theta_{i+1}\right)\right|_{\rho=\rho_{i+1}},\left.\quad \frac{d \theta_{i}}{d \rho}\right|_{\rho=\rho_{i+1}}=\left.K_{\lambda}^{(i+1)} \frac{d \theta_{i+1}}{d \rho}\right|_{\rho=\rho_{i+1}} \quad i=1,2 .
\end{gathered}
$$

In such manner, the original non-linear heat-conduction problems is reduced to the simpler one in terms of the Kirchhoff variable. In the latter problem, the non-linearity remains in (12), (13) and the first condition of (14). Due to the linear dependence of the heat-conduction coefficients on the temperature (5), formula (10) yields

$$
\theta_{i}=\left(T_{i}-T_{b}\right)+\frac{k_{i}}{2}\left(T_{i}-T_{b}\right)^{2} .
$$

From this equation we can find the following expression

$$
T_{i}\left(\theta_{i}\right)=\frac{\sqrt{1+2 k_{i} \theta_{i}}-1}{k_{i}}+T_{b} .
$$

Note that the sign of square root in the obtained expressions has to be chosen in the way to assure $T_{i}\left(\theta_{i}\right)$ having feasible meaning. 
By integrating equations (11), we arrive at

$$
\frac{d \theta_{i}}{d \rho}=\frac{C_{i 1}}{\rho}+\tilde{W}^{(i)} \delta_{i 2}, \quad \theta_{i}=C_{i 1} \ln \frac{\rho}{\rho_{i}}+C_{i 2}+\bar{W}^{(i)} \delta_{i 2}, \quad i=\overline{1,3},
$$

where

$$
\begin{aligned}
\tilde{W}^{(2)}(\rho)=- & \frac{4 P o}{\left(\rho_{3}-\rho_{2}\right)^{2}}\left(\frac{\rho^{3}}{4}-\frac{\rho^{2}}{3}\left(\rho_{3}+\rho_{2}\right)+\frac{\rho}{2} \rho_{3} \rho_{2}-\frac{\rho_{2}^{3}}{6 \rho}\left(\rho_{3}-\frac{\rho_{2}}{2}\right)\right) \\
\bar{W}^{(2)}(\rho)= & -\frac{4 P o}{\left(\rho_{3}-\rho_{2}\right)^{2}}\left(\rho^{2}\left(\frac{\rho^{2}}{16}-\frac{\rho}{9}\left(\rho_{3}+\rho_{2}\right)+\frac{\rho_{2} \rho_{3}}{4}\right)-\right. \\
& \left.-\rho_{2}^{3}\left(\frac{\rho_{2}}{16}-\frac{\rho_{3}+\rho_{2}}{9}+\frac{\rho_{3}}{4}\right)-\frac{\rho_{2}^{3}}{6} \ln \frac{\rho}{\rho_{2}}\left(\rho_{3}-\frac{\rho_{2}}{2}\right)\right) .
\end{aligned}
$$

The solutions (17) contain six unknown constants of integration. In order to find them, we assume the constants in the expression for Kirchhoff variables to be given (the basic ones) in the first layer $C_{11}$ and $C_{12}$, for example. Then the remaining constants can be expressed through the basic ones using the interface contact conditions (14). Note that hereby the condition [1]

$$
\left.\left(\theta_{i+1}-\theta_{i}\right)\right|_{z=z_{i+1}}=\left.\frac{k_{i+1}-k_{i}}{2}\left(T_{i}\left(\theta_{i}\right)-T_{p}\right)^{2}\right|_{z=z_{i+1}}, \quad i=1,2,
$$

has been equivalently used instead of the first one of (14). From the second of condition (14) we obtain

$$
C_{21}=\frac{C_{11}}{K_{\lambda}^{(2)}}, \quad C_{31}=\frac{1}{K_{\lambda}^{(3)}}\left(\frac{C_{11}}{K_{\lambda}^{(2)}}+\rho_{3} \tilde{W}^{(2)}\left(\rho_{3}\right)\right)
$$

meanwhile conditions (18) yield

$$
\begin{gathered}
C_{22}=C_{11} \ln \frac{\rho_{2}}{\rho_{1}}+C_{12}+\frac{k_{2}-k_{1}}{2}\left(\frac{\sqrt{1+2 k_{1}\left(C_{11} \ln \rho_{2} / \rho_{1}+C_{12}\right)}-1}{k_{1}}\right)^{2}, \\
C_{32}=\left.\theta_{2}\right|_{\rho=\rho_{3}}+\frac{k_{3}-k_{2}}{2}\left(\frac{\sqrt{1+\left.2 k_{1} \theta_{2}\right|_{\rho=\rho_{3}}}-1}{k_{2}}\right)^{2},
\end{gathered}
$$

where

$$
\left.\theta_{2}\right|_{\rho=\rho_{3}}=C_{11}\left(\ln \frac{\rho_{2}}{\rho_{1}}+\frac{1}{K_{\lambda}^{(2)}} \ln \frac{\rho_{3}}{\rho_{2}}\right)+C_{12}+\frac{k_{2}-k_{1}}{2}\left(\frac{\sqrt{1+2 k_{1}\left(C_{11} \ln \rho_{2} / \rho_{1}+C_{12}\right)}-1}{k_{1}}\right)^{2}+\bar{W}^{(2)}\left(\rho_{3}\right) .
$$

In such manner, the constant of integration $C_{i 1}$ та $C_{i 2} \quad(i=2,3)$ are expressed through the basic ones $C_{11}$ and $C_{12}$, which are found from boundary conditions (12)-(13). Using (12) we can determine the constant $C_{11}$

$$
C_{11}=\rho_{1}\left(B i_{1}\left(\frac{\sqrt{1+2 k_{1} C_{12}}-1}{k_{1}}+T_{b}-T_{i n s}\right)+S k_{1}\left(\left(\frac{\sqrt{1+2 k_{1} C_{12}}-1}{k_{1}}+T_{b}\right)^{4}-T_{i n s}^{4}\right)\right) .
$$

Formula (13), in turn, yields the algebraic equation

$$
\frac{C_{31}}{\rho_{4}}+B i_{3}\left(\frac{\sqrt{1+2 k_{3}\left(C_{31} \ln \rho_{4} / \rho_{3}+C_{32}\right)}-1}{k_{3}}+T_{b}-T_{\text {outs }}\right)=0
$$


for determination of constant $C_{12}$.

Due to the fact that equation (19) cannot be solved analytically, we employ the mathematical software for analytical manipulations for the specific input parameters. Having found $C_{12}$, we get the rest of constants automatically.

\section{Determination of the temperature distribution in a hollow nonthermosensitive cylin- der}

The dimensionless mathematical model for determining the temperature of a similarly formed, nonthermosensitive cylinder has the form

$$
\begin{gathered}
\frac{d}{d \rho}\left(\rho \frac{d T_{i n}}{d \rho}\right)=W_{n}^{(i)}(\rho) \delta_{2 i}, \quad \rho_{i}<\rho<\rho_{i+1}, \quad i=\overline{1,3} \\
{\left[\frac{d T_{1 n}}{d \rho}-B i_{1 n}\left(T_{1 n}-T_{i n s}\right)-S k_{1 n}\left(T_{1 n}^{4}-T_{i n s}^{4}\right)\right]_{\rho=\rho_{1}}=0,} \\
{\left[\frac{d T_{3 n}}{d \rho}+B i_{3 n}\left(T_{3 n}-T_{\text {outs }}\right)\right]_{\rho=\rho_{4}}=0,} \\
\left.T_{i n}\right|_{\rho=\rho_{i+1}}=\left.T_{(i+1) n}\right|_{\rho=\rho_{i+1}},\left.\quad \frac{d T_{i n}}{d \rho}\right|_{\rho=\rho_{i+1}}=\left.K_{\lambda}^{(i+1)} \frac{d T_{(i+1) n}}{d \rho}\right|_{\rho=\rho_{i+1}}, \quad i=1,2,
\end{gathered}
$$

where $W_{n}^{(2)}(\rho)=-4 P o_{n} \rho\left(\rho-\rho_{2}\right)\left(\rho-\rho_{3}\right) /\left(\rho_{3}-\rho_{2}\right)^{2}, P o_{n}=P o \lambda_{t 0}^{(2)} / \lambda_{t n}^{(2)}, B i_{i n}=B i_{i} \lambda_{t 0}^{(i)} / \lambda_{t n}^{(i)}(i=1,3)$, $S k_{1 n}=S k_{1} \lambda_{t 0}^{(1)} / \lambda_{t n}^{(1)}, K_{\lambda}^{(j)}=\lambda_{t n}^{(j)} / \lambda_{t n}^{(j-1)}(j=2,3)$.

From the problem $(20)-(23)$ we can find the following:

$$
\frac{d T_{i n}}{d \rho}=\frac{\bar{C}_{i 1}}{\rho}+\tilde{W}_{n}^{(i)}(\rho) \delta_{i 2}, \quad T_{i n}=\bar{C}_{i 1} \ln \frac{\rho}{\rho_{i}}+\bar{C}_{i 2}+\bar{W}_{n}^{(i)}(\rho) \delta_{i 2}, \quad i=\overline{1,3},
$$

where

$$
\begin{gathered}
\tilde{W}_{n}^{(2)}(\rho)=-\frac{4 P o_{n}}{\left(\rho_{3}-\rho_{2}\right)^{2}}\left(\frac{\rho^{3}}{4}-\frac{\rho^{2}}{3}\left(\rho_{3}+\rho_{2}\right)+\frac{\rho}{2} \rho_{3} \rho_{2}-\frac{\rho_{2}^{3}}{6 \rho}\left(\rho_{3}-\frac{\rho_{2}}{2}\right)\right), \\
\bar{W}_{n}^{(2)}(\rho)=-\frac{4 P o_{n}}{\left(\rho_{3}-\rho_{2}\right)^{2}}\left(\rho^{2}\left(\frac{\rho^{2}}{16}-\frac{\rho}{9}\left(\rho_{3}+\rho_{2}\right)+\frac{\rho_{2} \rho_{3}}{4}\right)-\right. \\
\left.-\rho_{2}^{3}\left(\frac{\rho_{2}}{16}-\frac{\rho_{3}+\rho_{2}}{9}+\frac{\rho_{3}}{4}\right)-\frac{\rho_{2}^{3}}{6} \ln \frac{\rho}{\rho_{2}}\left(\rho_{3}-\frac{\rho_{2}}{2}\right)\right) .
\end{gathered}
$$

Then, using the boundary conditions (23) we can find the constants of integration

$$
\begin{gathered}
\bar{C}_{21}=\frac{\bar{C}_{11}}{K_{\lambda}^{(2)}}, \quad \bar{C}_{22}=\bar{C}_{11} \ln \frac{\rho_{2}}{\rho_{1}}+\bar{C}_{12}, \\
\bar{C}_{31}=\frac{1}{K_{\lambda}^{(3)}}\left(\frac{\bar{C}_{11}}{K_{\lambda}^{(2)}}+\rho_{3} \tilde{W}^{(2)}\left(\rho_{3}\right)\right), \quad \bar{C}_{32}=\bar{C}_{11}\left(\ln \frac{\rho_{2}}{\rho_{1}}+\frac{1}{K_{\lambda}^{(2)}} \ln \frac{\rho_{3}}{\rho_{2}}\right)+\bar{C}_{12}+\bar{W}^{(2)}\left(\rho_{3}\right) .
\end{gathered}
$$

From the boundary conditions (21) we get the constant

$$
\bar{C}_{11}=\rho_{1}\left(B i_{1 n}\left(\bar{C}_{12}-T_{i n s}\right)+S k_{1 n}\left(\bar{C}_{12}^{4}-T_{i n s}^{4}\right)\right),
$$

Mathematical Modeling and Computing, Vol. 2, No. 2, pp. 160-175 (2015) 
meanwhile the condition (22) yields the equation

$$
\frac{\bar{C}_{31}}{\rho_{4}}+B i_{3 n}\left(\bar{C}_{31} \ln \frac{\rho_{4}}{\rho_{3}}+\bar{C}_{32}-T_{\text {outs }}\right)=0
$$

to determine the constant $\bar{C}_{12}$.

\section{Formulation of the mathematical model of stress-strain state}

Assume the layers of foregoing three-layered cylinder to be in the perfect mechanical contact and exposed to the determined radial distribution of temperature. The inner and outer cylindrical surfaces of the cylinder are subjected to uniformly distributed normal loadings. Assume the mechanical properties (the Young modulus $E^{(i)}\left(t_{i}\right)$, the Poisson ratio $\nu^{(i)}\left(t_{i}\right)$ and the linear thermal expansion coefficients $\left.\alpha_{t}^{(i)}\left(t_{i}\right)\right)$ of the layers to be functions of the form $\chi^{(i)}\left(t_{i}\right)=\chi_{0}^{(i)} \chi_{*}^{(i)}\left(T_{i}\right)$, where $\chi_{0}^{(i)}$ are the dimensional quantities equal to the value of the characteristic with the temperature $t_{b}$ and $\chi_{*}^{(i)}\left(T_{i}\right)$ are the dimensionless function of the dimensionless temperature $T_{i}$. Then,

$$
E^{(i)}\left(t_{i}\right)=E_{0}^{(i)} E_{*}^{(i)}\left(T_{i}\right), \quad \nu^{(i)}\left(t_{i}\right)=\nu_{0}^{(i)} \nu_{*}^{(i)}\left(T_{i}\right), \quad \alpha_{t}^{(i)}\left(t_{i}\right)=\alpha_{t 0}^{(i)} \alpha_{*}^{(i)}\left(T_{i}\right)
$$

The basic equations, which govern the stress-strain state of the cylinder, are:

- the equilibrium equations

$$
\frac{d}{d \rho}\left(\rho \sigma_{r}^{(i)}\right)=\rho \sigma^{(i)}, \quad i=\overline{1,3}
$$

- the physical strain-stress relations

$$
\begin{gathered}
E^{(i)} e_{r}^{(i)}=\sigma_{r}^{(i)}-\nu^{(i)}\left(\sigma_{\varphi}^{(i)}+\sigma_{z}^{(i)}\right)+E^{(i)} \Phi^{(i)}\left(T_{i}\right)= \\
=\left(1+\nu^{(i)}\right)\left(\sigma_{r}^{(i)}-\nu^{(i)} \sigma^{(i)}\right)-\nu^{(i)} E^{(i)} e_{z}+\left(1+\nu^{(i)}\right) E^{(i)} \Phi^{(i)}\left(T_{i}\right), \\
E^{(i)} e_{\varphi}^{(i)}=\sigma_{\varphi}^{(i)}-\nu^{(i)}\left(\sigma_{r}^{(i)}+\sigma_{z}^{(i)}\right)+E^{(i)} \Phi^{(i)}\left(T_{i}\right)= \\
=\left(1+\nu^{(i)}\right)\left(\left(1-\nu^{(i)}\right) \sigma^{(i)}-\sigma_{r}^{(i)}\right)-\nu^{(i)} E^{(i)} e_{z}+\left(1+\nu^{(i)}\right) E^{(i)} \Phi^{(i)}\left(T_{i}\right), \\
E^{(i)} e_{z}^{(i)}=\sigma_{z}^{(i)}-\nu^{(i)}\left(\sigma_{r}^{(i)}+\sigma_{\varphi}^{(i)}\right)+E^{(i)} \Phi^{(i)}\left(T_{i}\right)=\sigma_{z}^{(i)}-\nu^{(i)} \sigma^{(i)}+E^{(i)} \Phi^{(i)}\left(T_{i}\right), \quad i=\overline{1, n} ;
\end{gathered}
$$

- the compatibility equation

$$
\rho \frac{d e_{\varphi}^{(i)}}{d \rho}=e_{r}^{(i)}-e_{\varphi}^{(i)}
$$

that can be represented in terms of stresses

$$
\frac{d}{d \rho}\left(\frac{1-\left(\nu^{(i)}\right)^{2}}{E^{(i)}} \sigma^{(i)}-\nu^{(i)} e_{z}+\left(1+\nu^{(i)}\right) \Phi^{(i)}\left(T_{i}\right)\right)=\sigma_{r}^{(i)} \frac{d}{d \rho}\left(\frac{1+\nu^{(i)}}{E^{(i)}}\right) ;
$$

- the conditions on the limiting surfaces and in the cross section

$$
\sigma_{r}^{(1)}\left(\rho_{1}\right)=-p_{1}, \quad \sigma_{r}^{(n)}\left(\rho_{n+1}\right)=-p_{2}, \quad 2 \pi \int_{\rho_{1}}^{\rho_{n+1}} \nu \sigma_{z}(\nu) d \nu=p ;
$$

- the conditions of the perfect mechanical contact on the interfaces

$$
u_{r}^{(i+1)}\left(\rho_{i+1}\right)=u_{r}^{(i)}\left(\rho_{i+1}\right), \quad \sigma_{r}^{(i+1)}\left(\rho_{i+1}\right)=\sigma_{r}^{(i)}\left(\rho_{i+1}\right), \quad i=\overline{1, n-1} .
$$

Mathematical Modeling and Computing, Vol. 2, No. 2, pp. 160-175 (2015) 
Here, in $(25)-(31) \Phi^{(i)}\left(T_{i}\right)=t_{0} \int_{T_{b}}^{T_{i}} \alpha^{(i)}\left(T_{i}\right) d T_{i}$ is the purely thermal deformation; $\sigma_{r}^{(i)}, \sigma_{\varphi}^{(i)}, \sigma_{z}^{(i)}$ are the radial, circumferential and axial stresses; $e_{r}^{(i)}, e_{\varphi}^{(i)}$ are the radial and circumferential strains in $i$-layer, respectively; $e_{z}$ is the constant axial strain; $\sigma^{(i)}=\sigma_{r}^{(i)}+\sigma_{\varphi}^{(i)} ; u_{r}^{(i)}$ is the radial component of the displacement-vector in $i$-st layer related to the characteristic length $l_{0} ; p_{1}, p_{2}$ are the constant pressures on the inner $\left(\rho=\rho_{1}\right)$ and outer $\left(\rho=\rho_{n+1}\right)$ surfaces, respectively; $p$ is the axial loading at the ends of cylinder.

\section{Determination of the stress-strain state}

In [1], the following computation formulae have been obtained:

$$
\begin{gathered}
\sigma_{r}^{(i)}(\rho)=\gamma_{1 r}^{(i)}(\rho) \sigma^{(1)}\left(\rho_{1}\right)+\gamma_{2 r}^{(i)}(\rho) e_{z}+\gamma_{0 r}^{(i)}(\rho), \\
\sigma_{\varphi}^{(i)}(\rho)=\sigma^{(i)}(\rho)-\sigma_{r}^{(i)}(\rho), \\
\sigma_{z}^{(i)}(\rho)=E^{(i)} e_{z}+\nu^{(i)} \sigma^{(i)}(\rho)-E^{(i)} \Phi^{(i)}\left(T_{i}\right), \\
\sigma^{(i)}(\rho)=\gamma_{10}^{(i)}(\rho) \sigma^{(1)}\left(\rho_{1}\right)+\gamma_{20}^{(i)}(\rho) e_{z}+\gamma_{00}^{(i)}(\rho), \\
e_{r}^{(i)}(\rho)=\left(\sigma_{r}^{(i)}(\rho)-\nu^{(i)}\left(\sigma_{\varphi}^{(i)}(\rho)+\sigma_{z}^{(i)}(\rho)\right)+E^{(i)} \Phi^{(i)}\left(T_{i}\right)\right) / E^{(i)}, \\
e_{\varphi}^{(i)}(\rho)=\left(\sigma_{\varphi}^{(i)}(\rho)-\nu^{(i)}\left(\sigma_{r}^{(i)}(\rho)+\sigma_{z}^{(i)}(\rho)\right)+E^{(i)} \Phi^{(i)}\left(T_{i}\right)\right) / E^{(i)}, \\
u_{r}^{(i)}(\rho)=\rho \cdot e_{\varphi}^{(i)}(\rho),
\end{gathered}
$$

where

$$
\begin{aligned}
\gamma_{10}^{(i)}(\rho)= & \frac{1}{\psi^{(i)}(\rho)}\left(\left(1-\delta_{1 i}\right) \gamma_{1 r}^{(i-1)}\left(\rho_{i}\right) \chi_{2}^{(i)}(\rho)+\right. \\
& \left.+\left(\frac{1-\left(\nu^{(1)}\left(\rho_{1}\right)\right)^{2}}{E^{(1)}\left(\rho_{1}\right)}+\left(1-\delta_{1 i}\right) \sum_{k=1}^{i-1} \int_{\rho_{k}}^{\rho_{k+1}} \gamma_{1 r}^{(k)}(\eta)\left(\varphi^{(k)}(\eta)\right)^{\prime} d \eta+\beta^{(k)} \gamma_{1 r}^{(k)}\left(\rho_{k+1}\right)\right) \chi_{1}^{(i)}(\rho)\right), \\
\gamma_{20}^{(i)}(\rho)= & \frac{1}{\psi^{(i)}(\rho)}\left(\nu^{(i)}(\rho)-\nu^{(i)}\left(\rho_{i}\right)+\left(1-\delta_{1 i}\right) \gamma_{2 r}^{(i-1)}\left(\rho_{i}\right) \chi_{2}^{(i)}(\rho)+\right. \\
& \left.+\left(\nu^{(i)}\left(\rho_{i}\right)-\nu^{(1)}\left(\rho_{1}\right)+\left(1-\delta_{1 i}\right) \sum_{k=1}^{i-1}\left(\int_{\rho_{k}}^{\rho_{k+1}} \gamma_{2 r}^{(k)}(\eta)\left(\varphi^{(k)}(\eta)\right)^{\prime} d \eta+\beta^{(k)} \gamma_{2 r}^{(k)}\left(\rho_{k+1}\right)\right)\right) \chi_{1}^{(i)}(\rho)\right), \\
\gamma_{00}^{(i)}(\rho)= & \frac{1}{\psi^{(i)}(\rho)}\left(\left(-\delta_{1 i} p_{1}+\left(1-\delta_{1 i}\right) \gamma_{0 r}^{(i-1)}\left(\rho_{i}\right)\right) \chi_{2}^{(i)}(\rho)-F^{(i)}(\rho)+F^{(i)}\left(\rho_{i}\right)+\right. \\
& \left.+\left(\left(1-\delta_{1 i}\right) \sum_{k=1}^{i-1}\left(\int_{\rho_{k}}^{\rho_{k+1}} \gamma_{0 r}^{(k)}(\eta)\left(\varphi^{(k)}(\eta)\right)^{\prime} d \eta+\beta^{(k)} \gamma_{0 r}^{(k)}\left(\rho_{k+1}\right)\right)-F^{(i)}\left(\rho_{i}\right)\right) \chi_{1}^{(i)}(\rho)\right), \\
\gamma_{1 r}^{(i)}(\rho)= & \frac{1}{\rho^{2}}\left(\left(1-\delta_{1 i}\right) \rho_{i}^{2} \gamma_{1 r}^{(i-1)}\left(\rho_{i}\right)+\int_{\rho_{i}}^{\rho} \eta \gamma_{10}^{(i)}(\eta) d \eta\right)
\end{aligned}
$$




$$
\begin{aligned}
& \gamma_{2 r}^{(i)}(\rho)=\frac{1}{\rho^{2}}\left(\left(1-\delta_{1 i}\right) \rho_{i}^{2} \gamma_{2 r}^{(i-1)}\left(\rho_{i}\right)+\int_{\rho_{i}}^{\rho} \eta \gamma_{20}^{(i)}(\eta) d \eta\right), \\
& \gamma_{0 r}^{(i)}(\rho)=\frac{1}{\rho^{2}}\left(-\rho_{1}^{2} p_{1} \delta_{1 i}+\left(1-\delta_{1 i}\right) \rho_{i}^{2} \gamma_{0 r}^{(i-1)}\left(\rho_{i}\right)+\int_{\rho_{i}}^{\rho} \eta \gamma_{00}^{(i)}(\eta) d \eta\right), \\
& \chi_{1}^{(i)}=1+\left(\frac{\rho-\rho_{i}}{2}\right)^{2}\left(\varphi^{(i)}(\rho)\right)^{\prime} \frac{\rho_{i}}{\rho^{2}} \frac{E^{(i)}\left(\rho_{i}\right)}{1-\left(\nu^{(i)}\left(\rho_{i}\right)\right)^{2}}, \\
& \chi_{2}^{(i)}=\frac{\rho-\rho_{i}}{2}\left(\frac{\rho_{i}^{2}}{\rho^{2}}\left(\varphi^{(i)}(\rho)\right)^{\prime}+\left(\varphi^{(i)}\left(\rho_{i}\right)\right)^{\prime}\right), \\
& \psi^{(i)}(\rho)=\frac{1-\left(\nu^{(i)}(\rho)\right)^{2}}{E^{(i)}(\rho)}-\left(\frac{\rho-\rho_{i}}{2}\right)^{2} \frac{1}{\rho}\left(\varphi^{(i)}\left(\rho_{i}\right)\right)^{\prime}, \\
& \varphi^{(i)}(\rho)=\frac{1-\nu^{(i)}(\rho)}{E^{(i)}(\rho)}, \\
& \left(\varphi^{(i)}(\rho)\right)^{\prime}=\frac{d}{d \rho}\left(\frac{1-\nu^{(i)}(\rho)}{E^{(i)}(\rho)}\right) \\
& \beta^{(i)}=\varphi^{(i+1)}\left(\rho_{i+1}\right)-\varphi^{(i)}\left(\rho_{i+1}\right), \\
& F^{(i)}(\rho)=\left(1+\nu^{(i)}(\rho)\right) \Phi^{(i)}\left(T_{i}(\rho)\right)-\left(1+\nu^{(1)}\left(\rho_{1}\right)\right) \Phi^{(1)}\left(T_{1}\left(\rho_{1}\right)\right), \\
& \Phi^{(i)}\left(T_{i}\right)=t_{0} \int_{T_{b}}^{T_{i}} \alpha_{t}^{(i)}\left(T_{i}\right) d T_{i}, \\
& \sigma^{(1)}\left(\rho_{1}\right)=\frac{c_{1} d_{22}-c_{2} d_{12}}{d_{11} d_{22}-d_{21} d_{12}}, \quad e_{z}=\frac{c_{2} d_{11}-c_{1} d_{21}}{d_{11} d_{22}-d_{21} d_{12}}, \\
& d_{11}=\sum_{k=1}^{n} \int_{\rho_{k}}^{\rho_{k+1}} \eta \gamma_{10}^{(k)}(\eta) d \eta \\
& d_{12}=\sum_{k=1}^{n} \int_{\rho_{k}}^{\rho_{k+1}} \eta \gamma_{20}^{(k)}(\eta) d \eta \\
& d_{21}=\sum_{k=1}^{n} \int_{\rho_{k}}^{\rho_{k+1}} \eta \nu^{(k)}(\eta) \gamma_{10}^{(k)}(\eta) d \eta \\
& d_{22}=\sum_{k=1}^{n} \int_{\rho_{k}}^{\rho_{k+1}} \eta\left(\nu^{(k)}(\eta) \gamma_{20}^{(k)}(\eta)+E^{(k)}(\eta)\right) d \eta \\
& c_{1}=\rho_{1}^{2} p_{1}-\rho_{n+1}^{2} p_{2}+\sum_{k=1}^{n} \int_{\rho_{k}}^{\rho_{k+1}} \eta \gamma_{00}^{(k)}(\eta) d \eta \\
& c_{2}=\frac{p}{2 \pi}+\sum_{k=1}^{n} \int_{\rho_{k}}^{\rho_{k+1}} \eta\left(E^{(k)}(\eta) \Phi^{(k)}\left(T_{k}(\eta)\right)-\nu^{(k)}(\eta) \gamma_{00}^{(k)}(\eta)\right) d \eta
\end{aligned}
$$


Note that formulae (32)-(35) are valid for the thin layers only, for which, then computing the integrals that contain unknown stresses, the trapezium rule

$$
\int_{\rho_{i}}^{\rho} Y(\eta) d \eta=\frac{\rho-\rho_{i}}{2}\left(Y(\rho)+Y\left(\rho_{i}\right)\right)
$$

is satisfied within the given accuracy.

If the cylinder contains both thin and thick layers, then each of the thick layers can be segmented into several thinner ones made of the same material. The accuracy of such representation can be verified by making use of the formula [1]

$$
\rho_{1}^{2} p_{1}-\rho_{n+1}^{2} p_{2}=\sum_{k=1}^{n} \int_{\rho_{k}}^{\rho_{k+1}} \eta \sigma^{(k)}(\eta) d \eta .
$$

\section{Special cases}

Case 1. Consider the case when the thermo-physical and mechanical characteristics of the nonthermosensitive cylinder (denote them with $n$ ) are equal to the basic values of corresponding components of the thermosensitive one, that are the values of thermosensitive characteristics at the temperature $t_{b}$. Then, the temperature distribution has the form (24), where $\lambda_{t n}^{(i)}=\lambda_{t 0}^{(i)}, i=\overline{1,3}$. The Poisson ratio, the coefficient of linear thermal expansion and the Young modulus have the following form: $\nu_{n}^{(i)}=\nu_{0}^{(i)}$, $\alpha_{t n}^{(i)}=\alpha_{t 0}^{(i)}, E_{n}^{(i)}=E_{0}^{(i)}, i=\overline{1,3}$, and formulae for determination of the the stress-strain state take the form

$$
\begin{gathered}
\sigma_{n}^{(i)}(\rho)=\gamma_{10 n}^{(i)}(\rho) \sigma_{n}^{(1)}\left(\rho_{1}\right)+\gamma_{20 n}^{(i)}(\rho) e_{z n}+\gamma_{00 n}^{(i)}(\rho), \\
\sigma_{r n}^{(i)}(\rho)=\gamma_{1 r n}^{(i)}(\rho) \sigma_{n}^{(1)}\left(\rho_{1}\right)+\gamma_{2 r n}^{(i)}(\rho) e_{z n}+\gamma_{0 r n}^{(i)}(\rho), \\
\sigma_{\varphi n}^{(i)}(\rho)=\sigma_{n}^{(i)}(\rho)-\sigma_{r n}^{(i)}(\rho), \\
\sigma_{z n}^{(i)}(\rho)=E_{0}^{(i)} e_{z n}+\nu_{0}^{(i)} \sigma_{n}^{(i)}(\rho)-E_{0}^{(i)} \Phi_{n}^{(i)}\left(T_{i n}\right), \\
e_{r n}^{(i)}(\rho)=\left(\sigma_{r n}^{(i)}(\rho)-\nu_{0}^{(i)}\left(\sigma_{\varphi n}^{(i)}(\rho)+\sigma_{z n}^{(i)}(\rho)\right)+E_{0}^{(i)} \Phi_{n}^{(i)}\left(T_{i n}\right)\right) / E_{0}^{(i)}, \\
e_{\varphi n}^{(i)}(\rho)=\left(\sigma_{\varphi n}^{(i)}(\rho)-\nu_{0}^{(i)}\left(\sigma_{r n}^{(i)}(\rho)+\sigma_{z n}^{(i)}(\rho)\right)+E_{0}^{(i)} \Phi_{n}^{(i)}\left(T_{i n}\right)\right) / E_{0}^{(i)}, \\
u_{r n}^{(i)}(\rho)=\rho \cdot e_{\varphi n}^{(i)}(\rho),
\end{gathered}
$$

where

$$
\begin{aligned}
& \gamma_{10 n}^{(i)}(\rho)=\frac{1}{\psi_{n}^{(i)}}\left(\frac{1-\left(\nu_{0}^{(i)}\right)^{2}}{E_{0}^{(i)}}+\left(1-\delta_{1 i}\right) \sum_{k=1}^{i-1} \beta_{n}^{(k)} \gamma_{1 r n}^{(k)}\left(\rho_{k+1}\right)\right) \\
& \gamma_{10 n}^{(i)}(\rho)=\frac{1}{\psi_{n}^{(i)}}\left(\nu_{0}^{(i)}-\nu_{0}^{(1)}+\left(1-\delta_{1 i}\right) \sum_{k=1}^{i-1} \beta_{n}^{(k)} \gamma_{2 r n}^{(k)}\left(\rho_{k+1}\right)\right) \\
& \gamma_{1 r n}^{(i)}(\rho)=\frac{1}{\rho^{2}}\left(\left(1-\delta_{1 i}\right) \rho_{i}^{2} \gamma_{1 r n}^{(i-1)}\left(\rho_{i}\right)+\int_{\rho_{i}}^{\rho} \eta \gamma_{10 n}^{(i)} d \eta\right)
\end{aligned}
$$

Mathematical Modeling and Computing, Vol. 2, No. 2, pp. 160-175 (2015) 


$$
\begin{aligned}
& \gamma_{2 r n}^{(i)}(\rho)=\frac{1}{\rho^{2}}\left(\left(1-\delta_{1 i}\right) \rho_{i}^{2} \gamma_{2 r n}^{(i-1)}\left(\rho_{i}\right)+\int_{\rho_{i}}^{\rho} \eta \gamma_{20 n}^{(i)} d \eta\right), \\
& \gamma_{0 r n}^{(i)}(\rho)=\frac{1}{\rho^{2}}\left(-\rho_{1}^{2} p_{1} \delta_{1 i}+\left(1-\delta_{1 i}\right) \rho_{i}^{2} \gamma_{0 r n}^{(i-1)}\left(\rho_{i}\right)+\int_{\rho_{i}}^{\rho} \eta \gamma_{00 n}^{(i)} d \eta\right), \\
& \psi_{n}^{(i)}=\frac{1-\left(\nu_{0}^{(i)}\right)^{2}}{E_{0}^{(i)}} \\
& \varphi_{n}^{(i)}=\frac{1-\nu_{0}^{(i)}}{E_{0}^{(i)}} \\
& \beta_{n}^{(i)}=\varphi_{n}^{(i+1)}-\varphi_{n}^{(i)}, \\
& \Phi_{n}^{(i)}\left(T_{i n}\right)=t_{0} \alpha_{t 0}^{(i)}\left(T_{i n}-T_{b}\right), \\
& F_{n}^{(i)}(\rho)=\left(1+\nu_{0}^{(i)}\right) \Phi_{n}^{(i)}\left(T_{i n}(\rho)\right)-\left(1+\nu_{0}^{(1)}\right) \Phi_{n}^{(1)}\left(T_{1 n}\left(\rho_{1}\right)\right), \\
& \sigma_{n}^{(1)}\left(\rho_{1}\right)=\frac{c_{1 n} d_{22 n}-c_{2 n} d_{12 n}}{d_{11 n} d_{22 n}-d_{21 n} d_{12 n}} \\
& e_{z n}=\frac{c_{2 n} d_{11 n}-c_{1 n} d_{21 n}}{d_{11 n} d_{22 n}-d_{21 n} d_{12 n}}, \\
& d_{11 n}=\sum_{k=1}^{n} \int_{\rho_{k}}^{\rho_{k+1}} \eta \gamma_{10 n}^{(k)}(\eta) d \eta \\
& d_{12 n}=\sum_{k=1}^{n} \int_{\rho_{k}}^{\rho_{k+1}} \eta \gamma_{20 n}^{(k)}(\eta) d \eta \\
& d_{21 n}=\sum_{k=1}^{n} \int_{\rho_{k}}^{\rho_{k+1}} \eta \nu_{0}^{(k)} \gamma_{10 n}^{(k)}(\eta) d \eta \\
& d_{22 n}=\sum_{k=1}^{n} \int_{\rho_{k}}^{\rho_{k+1}} \eta\left(\nu_{0}^{(k)} \gamma_{20 n}^{(k)}(\eta)+E_{0}^{(k)}\right) d \eta \\
& c_{1 n}=\rho_{1}^{2} p_{1}-\rho_{n+1}^{2} p_{2}+\sum_{k=1}^{n} \int_{\rho_{k}}^{\rho_{k+1}} \eta \gamma_{00 n}^{(k)}(\eta) d \eta, \\
& c_{2 n}=\frac{p}{2 \pi}+\sum_{k=1}^{n} \int_{\rho_{k}}^{\rho_{k+1}} \eta\left(E_{0}^{(k)} \Phi^{(k)}\left(T_{k}(\eta)\right)-\nu_{0}^{(k)} \gamma_{00 n}^{(k)}(\eta)\right) d \eta .
\end{aligned}
$$

Case 2. In case of the average integral values of heat-conduction coefficients

$$
\begin{gathered}
\lambda_{t n}^{(i)}=\lambda_{t c}^{(i)}=\frac{1}{T_{e}-T_{b}} \int_{T_{b}}^{T_{e}} \lambda_{t 0}^{(i)}\left(1+k_{i}\left(T_{i}-T_{b}\right)\right) d T_{i}=\lambda_{t 0}^{(i)}\left(\frac{k_{i}}{2}\left(T_{e}-T_{b}\right)\right), \quad i=\overline{1,3} \\
\left\{\nu_{n}^{(i)}, \alpha_{t n}^{(i)}, E_{n}^{(i)}\right\}=\left\{\nu_{c}^{(i)}, \alpha_{t c}^{(i)}, E_{c}^{(i)}\right\}=\frac{1}{T_{e}-T_{b}} \int_{T_{b}}^{T_{e}}\left\{\nu_{c}^{(i)}, \alpha_{t c}^{(i)}, E_{c}^{(i)}\right\} d T_{i}, \quad i=\overline{1,3}
\end{gathered}
$$


we obtain the temperature distribution (24). The formulae for calculation of the stress-strain state will have the form (41)-(47) in which the basic values of the thermomechanical characteristics should be replaced by average integral ones.

\section{Numerical implementation}

We intend to analyze the thermosensitivity effect in the distribution of temperature and stress-strain components in the three-layer cylinder for different parameters. Assume the first and third layers to be made of ceramics $\mathrm{ZrO}_{2}$, meanwhile the second one is made of the titanium alloy $\mathrm{Ti}-6 \mathrm{Al}-4 \mathrm{~V}$ [6].

Approximate the experimentally found heat-conduction coefficients and thermomechanical characteristics by polynomials (5) within the temperature range $300 \div 1100$ using the least squares method. The following values were established for ceramics

$$
\begin{aligned}
& \lambda_{t}^{(1,3)}=1.915\left(1+0.24664\left(T_{1,3}-T_{b}\right)\right)[W /(m \cdot K)], \quad \nu^{(1,3)}=0.333, \\
& \alpha^{(1,3)}=8.783 \cdot 10^{-6}\left(1-1.4128\left(T_{1,3}-T_{b}\right)+1.7496\left(T_{1,3}-T_{b}\right)^{2}\right)[1 / K], \\
& E^{(1,3)}=116.381\left(1-0.521357\left(T_{1,3}-T_{b}\right)-0.084215\left(T_{1,3}-T_{b}\right)^{2}\right)[G P a],
\end{aligned}
$$

and for titanium alloy

$$
\begin{aligned}
& \lambda_{t}^{(2)}=6.2\left(1+3.016\left(T_{2}-T_{b}\right)\right)[W /(m \cdot K)], \quad \nu^{(1,3)}=0.2984\left(1+0.118\left(T_{2}-T_{b}\right)\right), \\
& \alpha^{(2)}=8.8559 \cdot 10^{-6}\left(1+0.49014\left(T_{2}-T_{b}\right)-0.36754\left(T_{2}-T_{b}\right)^{2}\right)[1 / K] \\
& E^{(2)}=105.05\left(1-0.5916\left(T_{2}-T_{b}\right)[G P a],\right.
\end{aligned}
$$

Impose $\rho_{1}=0.7, \rho_{2}=0.75, \rho_{3}=0.95, \rho_{4}=1$, and $l_{0}=r_{4}$. The dimensionless values of the temperature are assumed to be $T_{b}=3 / 11, T_{e}=1, T_{\text {ins }}=1 / 3, T_{\text {outs }}=1 / 3$, and the Pomerantsev, Biot and Stark numbers are $P o=-3, B i_{1}=1, B i_{2}=2, S k_{1}=0.5$. The cylinder is free of the pressures on the inner and outer surfaces and axial loadings at the end-faces $\left(p_{1}=p_{2}=p=0\right)$.

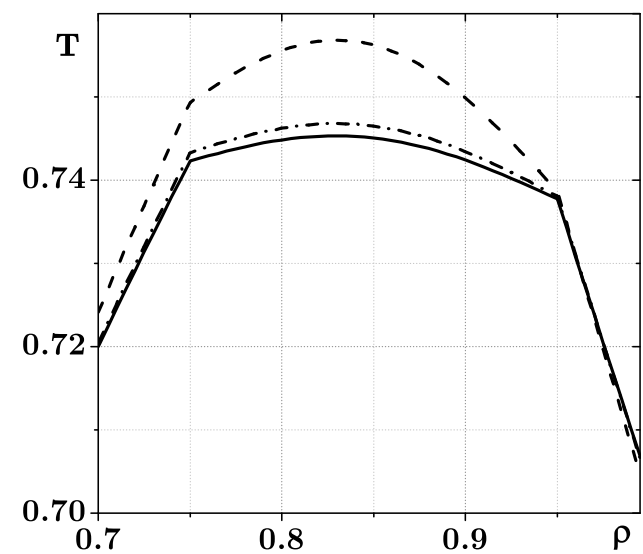

Fig. 1. The temperature distribution.

The temperature distributions for the considered cylinder are presented in Fig.1. Here and in what follow, the solid lines correspond to the thermosensitive cylinder, dashed lines correspond to the non-thermosensitive cylinder with basic values, dash-dotted lines correspond to the nonthermosensitive cylinder with the average integral values of the heat-conduction coefficient.

The maximum difference between the temperatures in thermosensitive and non-thermosensitive (with basic and average integral values of the coefficient of thermal conductivity) cylinders do not exceed $1 \%$

The algorithm for determining the components of stressstrain state, because the formulas (32)-(35) are valid for thin layers only, implies the following steps:

1. Compute the formulae (32)-(35) without segmentation of each layer into thin sublayers;

2. Verify the integral condition (40);

3. If the condition (40) is not satisfied, then increase the number of segments and return to step 2. Otherwise, the computation is over.

Tab.1 presents the results obtained when finding the sufficient segmentation of layers into sublayers in order to achieve the accuracy $\varepsilon=10^{-4}$. 
Table 1. The result of segmentation of cylinder layers into sublayers

\begin{tabular}{|c|c|c|c|c|}
\hline \multirow{2}{*}{$\begin{array}{c}\text { Total number } \\
\text { of layers }\end{array}$} & \multicolumn{2}{|c|}{ Number of sublayers in each layer } & $\begin{array}{c}\text { Verification of } \\
\text { integral conditions }\end{array}$ \\
\hline 3 & I layer & II layer & III layer & -0.000714 \\
\hline 4 & 1 & 1 & 1 & -0.000166 \\
\hline 5 & 1 & 2 & 1 & -0.000078 \\
\hline 6 & 1 & 3 & 1 & -0.000048 \\
\hline
\end{tabular}

As we can see the representation of the considered cylinder by a six-layer body is enough to achieve the required accuracy. The corresponding distributions of stresses, strains and displacements are shown in Fig.2-4. Note, that the constant axial strain equals 0.0044256.
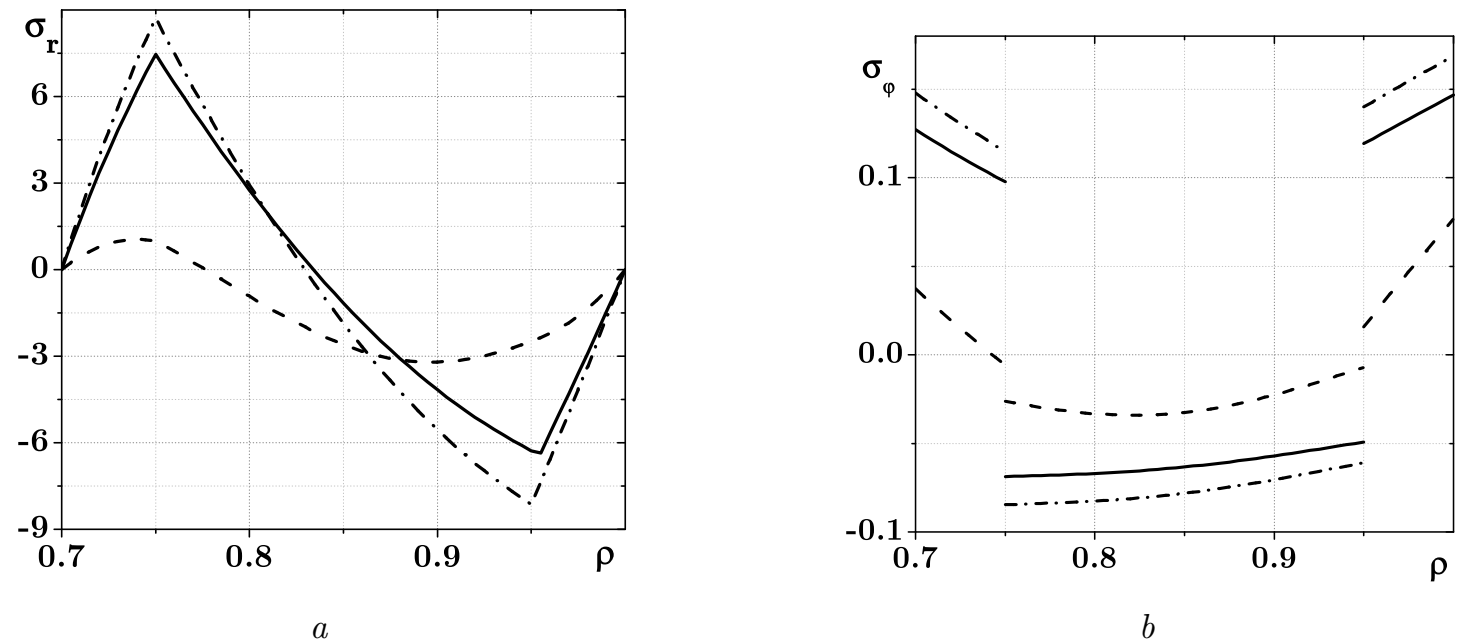

$a$

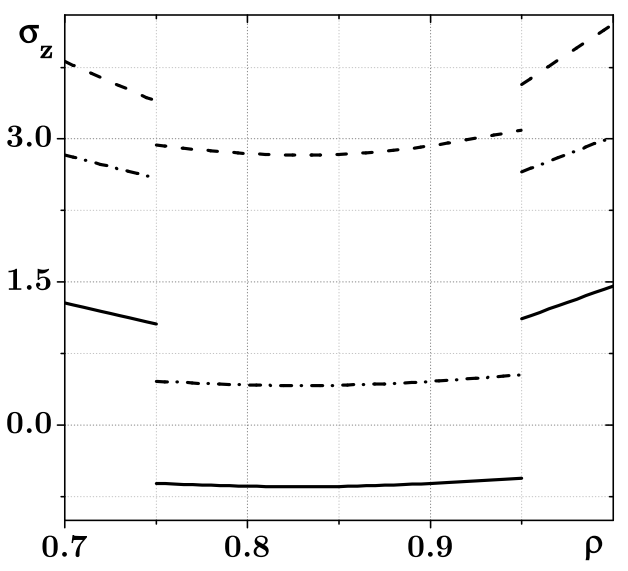

$c$

Fig. 2. Distributions of radial $\sigma_{r}(\rho) \cdot 10^{3}$ (a), circumferential $\sigma_{\varphi}(\rho)$ (b) and axial $\sigma_{z}(\rho) \cdot 10$ (c) stresses.

In Fig. 2-4, we can observe that the distribution of the stress-strain components in the nonthermosensitive cylinder with average integral values of mechanical characteristics gives a better approximation to the same distribution in thermosensitive hollow cylinder, in comparison to the stress-strain components distribution in the nonthermosensitive cylinder with basic values. In particular, the maximum differences are $25 \%$ for the radial stress, $30 \%$ for the circumferential stress, $30 \%$ for the radial strain, $10 \%$ for the circumferential strain, and $10 \%$ for the radial displacement.

The employment of the model of nonthermosensitive body with basic values of its thermomechanical characteristics does not provide a correct approximation for such distribution of appropriate stress- 


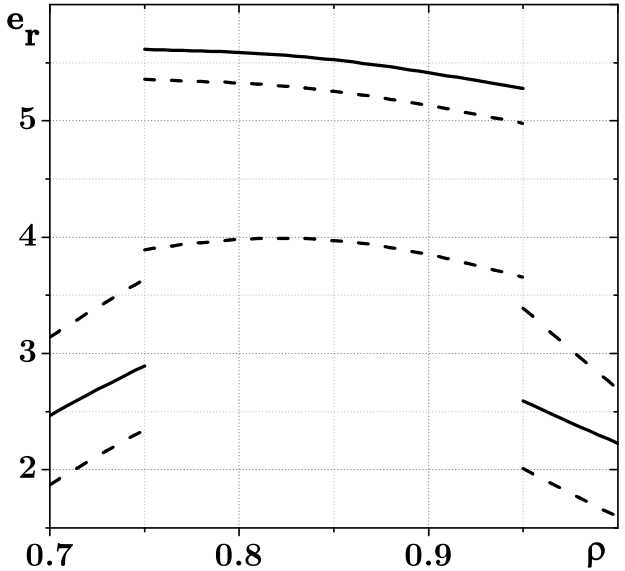

$a$

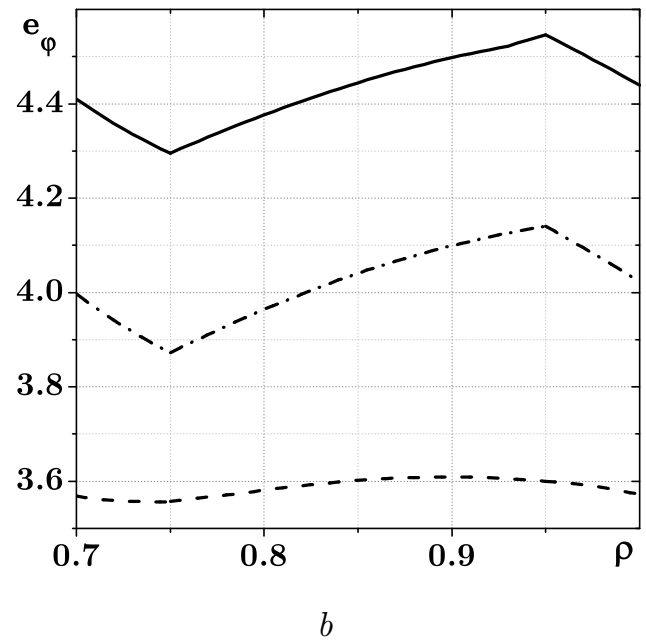

Fig. 3. Distributions of radial $e_{r}(\rho) \cdot 10^{3}$ (a) and circumferential $e_{\varphi}(\rho) \cdot 10^{3}$ (b) deformations.

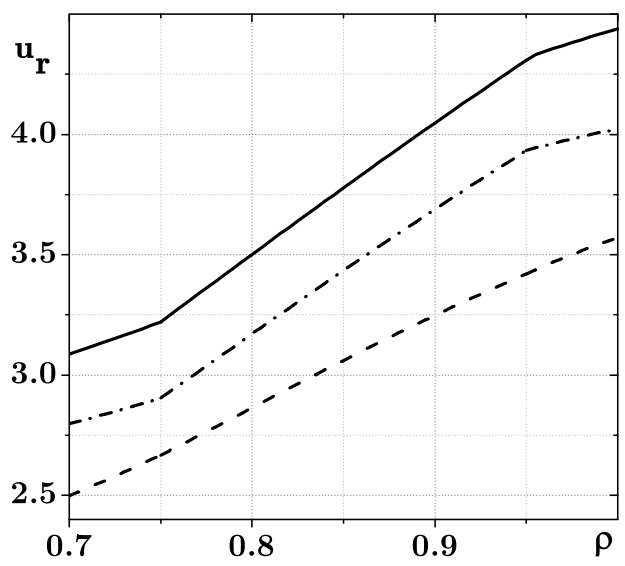

Fig. 4. Distributions of radial displacement $u_{r}(\rho) \cdot 10^{3}$.

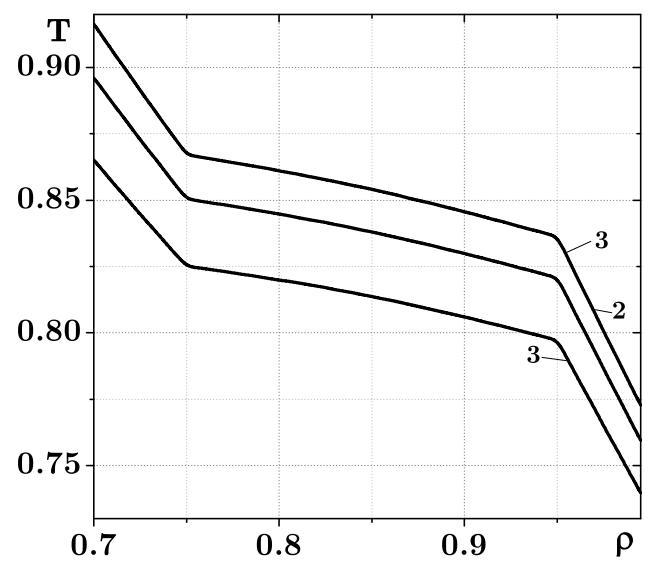

Fig. 5. The temperature distribution.

strain components in thermosensitive cylinder. Moreover, it leads to the qualitatively different results in some cases. In Fig. $2 c$, we can observe that the axial stress in the second layer of nonthermosensitive cylinder (with basic and average integral thermomechanical characteristics) are changed not only quantitatively, but also inherently (the compression in the thermosensitive cylinder and tension in the nonthermosensitive one). Therefore, it is necessary to take into account the thermosensitivity of material components to determine axial stresses.

The influence of the radiative heat exchange on the distribution of the temperature and stress strain state of the thermosensitive hollow cylinder has been investigated for the same coordinates of layer interfaces and material properties. The dimensionless temperatures were given as $T_{\text {ins }}=1, T_{\text {outs }}=$ $=5 / 11$ so that they were higher than the temperature of the cylinder. The dimensionless parameters are $P o=-2, B i_{1}=7, B i_{3}=5, S k_{1}=\{0,1,2\}$. Note, that there is the pure convective heat exchange on the inner limiting surface, if $S k_{1}=0$. In Fig. 5-8, the distributions of temperatures and stress-strain state components are shown. Here, lines 1 correspond to $S k_{1}=0,2-S k_{1}=1,3-S k_{1}=2$.

It is shown that under the imposed conditions, the increment in the Stark number by one unit causes the increment of the temperature and stress-strain state components more than i $6 \%$. But, if temperatures, radial displacements and axial strains increase almost uniformly with the change of $S k_{1}$ at each point of the cylinder, the radial, circumferential and axial stresses change mainly at the interfaces. 


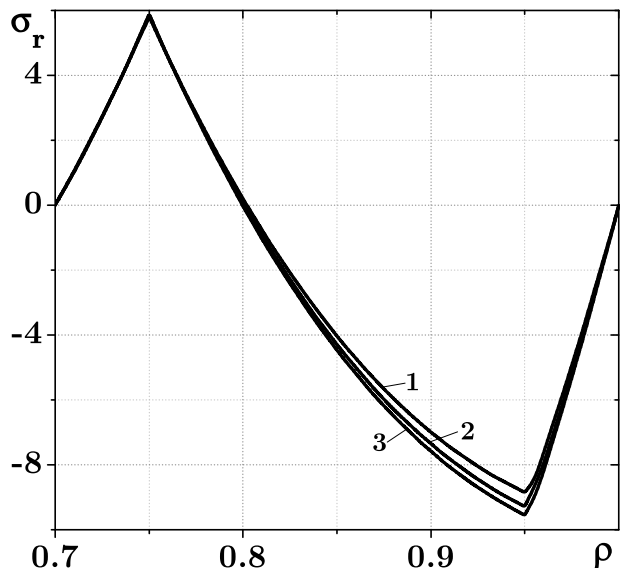

$a$

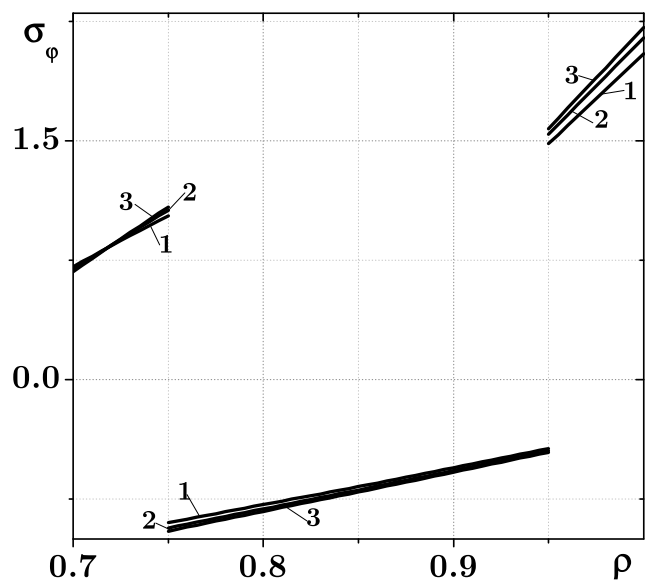

$b$

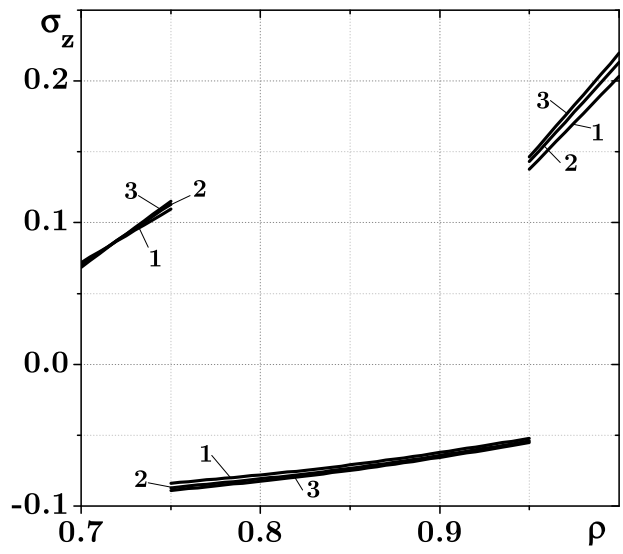

$c$

Fig. 6. Distributions of the radial $\sigma_{r}(\rho) \cdot 10^{3}(\mathrm{a})$, circumferential $\sigma_{\varphi}(\rho) \cdot 10(\mathrm{~b})$ and axial $\sigma_{z}(\rho) \cdot 10(\mathrm{c})$ stresses.

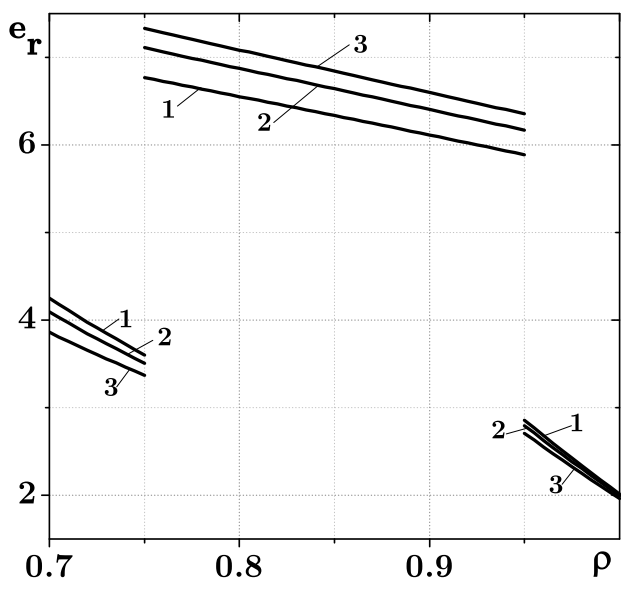

$a$

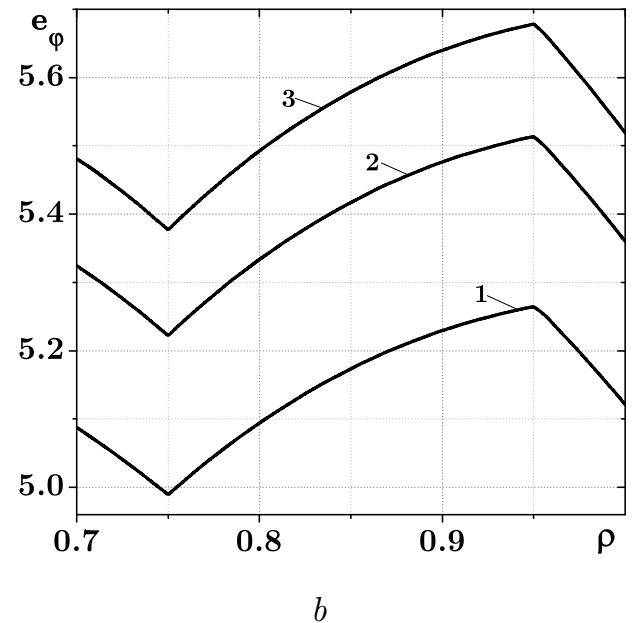

Fig. 7. Distributions of the radial $e_{r}(\rho) \cdot 10^{3}$ (a) and circumferential $e_{\varphi}(\rho) \cdot 10^{3}$ (b) strains. 


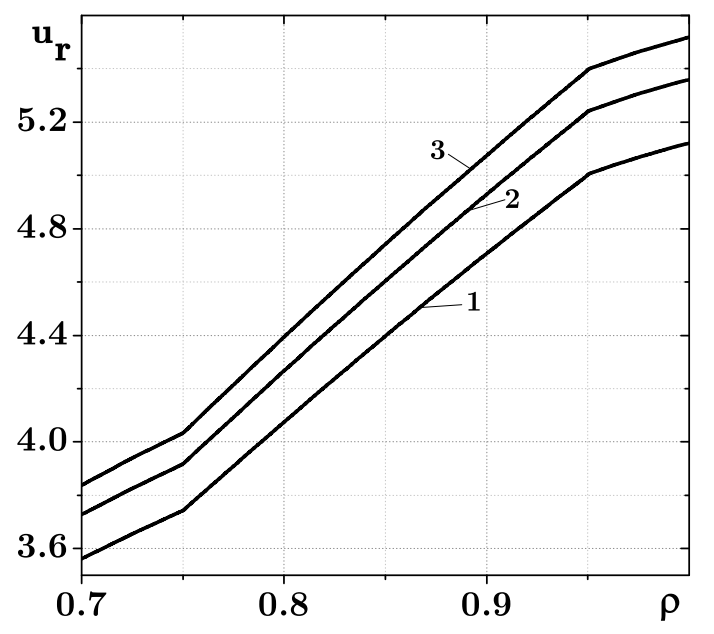

Fig. 8. Distributions of the radial displacement $u_{r}(\rho) \cdot 10^{3}$.

\section{Conclusions}

The nonlinear mathematical model of temperature distribution in an infinitely-long three-layer hollow thermosensitive cylinder with heat sources distributed by parabolic law in the second layer under the convective-radiative and convective heat exchange on the boundary surfaces is suggested. On this basis, the temperature and components of the stress-strain state are determined.

The thermosensitivity effect on the temperature and stress-strain state components distribution is analyzed. It is shown that neglecting the dependence of thermomechanical characteristics (the replacement for basic or average integral ones) causes the significant differences between the distributions of stress-strain state components in the thermosensitive and nonthermosensitive cylinders, which may lead to qualitatively different distributions (the compression in the thermosensitive cylinder and stretching in nonthermosensitive one).

For the considered materials, it is shown that the distribution of the stress-strain state in nonthermosensitive cylinder with average integral values of mechanical characteristics gives a better approximation to the corresponding distribution in the thermosensitive hollow cylinder. This can be useful for its quick estimation.

The investigation of the intensity of radiative heat exchange shows that the increment in the Stark number causes, in our case, the increment of the temperature and stress-strain state components in each point of cylinder.

[1] Popovych V., Kalyniak B. The mathematical modelling and method of determination of static thermostressed state of the multilayer thermosensitive cylinders (in Ukrainian). The mathematical metods and physical and machanical fields. 57, 2 (2014).

[2] Carslaw H., Jaeger J. Conduction of heat in solids, 2nd ed. Oxford, Clarendon Press (1959).

[3] Kushnir R., Popovych V. Thermoelasticity of Thermosensitive Solids. Lviv, SPOLOM (2009) (in Ukrainian).

[4] Kushnir R., Popovych V.S. Heat conduction problem of the thermosensitive solids onder complex heat exchange. Heat conduction - Basic research, In Tech (2011).

[5] Noda N. Thermal stresses in materials with temperature-dependent properties. Thermal stresses I. (1986).

[6] Tanigava Y., Akai T. Transient heat conduction and thermal stress problems of a nonhomogegenous plate with temperature dependent material properties. Journal of thermal stresses. 19, 1 (1996). 


\title{
Математичне моделювання, визначення та дослідження термопружного стану термочутливого тришарового порожнистого циліндра за конвективно-променевого теплообміну
}

\author{
Кушнір Р. M. ${ }^{1}$, Попович В. С. ${ }^{1}$, Ракоча I. I. ${ }^{2}$ \\ ${ }^{1}$ Інститут прикладних проблем механіки $і$ математики \\ ім. Я. С. Підстригача НАН України \\ вул. Наукова, 3б, т9060, Львів, Україна \\ ${ }^{2}$ Національний університет «Львівсъка політехніка» \\ вул. С. Бандери, 12, 79013, Лъвів, Україна
}

\begin{abstract}
Змодельовано та визначено стаціонарний розподіл температури в тришаровому безмежному порожнистому циліндрі, в другому шарі якого наявні джерела тепла, розподілені за параболічним законом, а на внутрішній та зовнішній обмежувальних поверхнях відбуваються конвективно-променевий та конвективний теплообміни відповідно. Знайдено компоненти напружено-деформованого стану. Досліджено вплив залежності теплових та механічних характеристик матеріалів складових від температури, а також інтенсивності променевого теплообміну на величину та характер розподілу температури і компонент напружено-деформованого стану.
\end{abstract}

Ключові слова: кусково однорідний ииліндр, порожнистий ииліндр, термочутливий матеріал, температура, термальна напруженість

2000 MSC: $97 \mathrm{M} 50$

удк: 593.3 